\title{
Protective Effects of Quercetin on Livers from Mice Exposed to Long-Term Cigarette Smoke
}

\author{
Pedro A. Machado-Junior $\mathbb{D}^{1},{ }^{1}$ Natália P. S. Araújo $\mathbb{D}^{1}{ }^{1}$ Ana B. F. Souza $\left(\mathbb{D},{ }^{1}\right.$ \\ Thalles F. Castro $\mathbb{D},{ }^{1}$ Michel Oliveira $\mathbb{D}^{1},{ }^{1}$ Guilherme P. Costa $\mathbb{D}^{2},{ }^{2}$ Natália A. Matos $\mathbb{D}^{1}{ }^{1}$ \\ Paula M. A. Vieira $\mathbb{D}^{D},{ }^{3}$ André Talvani $\mathbb{D}^{D},{ }^{2}$ Frank S. Bezerra $\mathbb{D}^{1},{ }^{1}$ and Sílvia D. Cangussú $\mathbb{D}^{1}$ \\ ${ }^{1}$ Laboratory of Experimental Pathophysiology (LAFEx), Department of Biological Sciences (DECBI), Institute of Exact and Biological \\ Sciences (ICEB), Federal University of Ouro Preto (UFOP), 35400-000 Ouro Preto, MG, Brazil \\ ${ }^{2}$ Laboratory of Immunobiology of Inflammation (LABIIN), Department of Biological Sciences (DECBI), Institute of Exact and \\ Biological Sciences (ICEB), Federal University of Ouro Preto (UFOP), 35400-000 Ouro Preto, MG, Brazil \\ ${ }^{3}$ Laboratory of Morphopathology (LM), Department of Biological Sciences (DECBI), Institute of Exact and Biological Sciences (ICEB), \\ Federal University of Ouro Preto (UFOP), 35400-000 Ouro Preto, MG, Brazil
}

Correspondence should be addressed to Sílvia D. Cangussú; cangussu@ufop.edu.br

Received 17 June 2020; Revised 15 October 2020; Accepted 19 October 2020; Published 17 November 2020

Academic Editor: Abdelwahab Omri

Copyright (c) 2020 Pedro A. Machado-Junior et al. This is an open access article distributed under the Creative Commons Attribution License, which permits unrestricted use, distribution, and reproduction in any medium, provided the original work is properly cited.

\begin{abstract}
Cigarette smoke is highly toxic, and it can promote increased production of reactive species and inflammatory response and leads to liver diseases. Quercetin is a flavonoid that displays antioxidant and anti-inflammatory activities in liver diseases. This study aimed at evaluating the protective effects of quercetin on livers from mice exposed to long-term cigarette smoke exposure. Male C57BL/6 mice were divided into five groups: control (CG), vehicle (VG), quercetin (QG), cigarette smoke (CSG), quercetin, and cigarette smoke (QCSG). CSG and QCSG were exposed to cigarette smoke for sixty consecutive days; at the end of the exposures, all animals were euthanized. Mice that received quercetin daily and were exposed to cigarette smoke showed a reduced influx of inflammatory cells, oxidative stress, inflammatory reaction, and histopathological changes in the liver, compared to CSG. These results suggest that quercetin may be an effective adjuvant for treating damage to the liver due to cigarette smoke exposure.
\end{abstract}

\section{Introduction}

Cigarette smoking is considered one of the leading preventable causes of morbidity and mortality representing a risk factor for several diseases such as cardiovascular, chronic obstructive pulmonary, and liver diseases [1,2]. Smoking kills about 6 million people a year, and it is estimated that this number will reach 8 million by 2030. Cigarette smoke (CS) is a highly toxic complex mixture of components containing more than 5000 compounds that can promote damage to biological tissues [3].

The CS produces reactive oxygen species (ROS) that, at high concentrations, brings about changes in the redox process and induction of inflammatory responses, triggering several comorbidities [4-6]. The exposure to cigarette smoke causes damage to cells and tissues by developing oxidative processes. The installation of oxidative stress can occur directly through the production of ROS or by the activation of the inflammatory process, which, in turn, results in an increased level of radical species $[7,8]$.

The liver is the biggest gland of the body, which is responsible for processing drugs, alcohol, and other toxins. As an immunologically complex organ, the liver contains multiple types of tissue-resident inflammatory cells, like lymphocytes [9] and Kupffer cells [10]. Therefore, liver diseases represent an important public health problem, and understanding the mechanisms of the pathophysiological process is crucial. The redox imbalance induced by chronic exposure to cigarette smoke produces toxins with direct or indirect toxic effects in the liver, promoting an increase in the number 
of inflammatory cells, collagen deposition, and apoptosis. Moreover, constituents of cigarette smoke exert immunoregulatory actions and hepatic carcinogens [11].

Previous studies showed that anti-inflammatory and antioxidant activities in natural compounds prevented diseases. In this context, it is worth to highlight the role of quercetin, which is the most abundant flavonol, and it is present in a variety of foodstuffs like fruits and vegetables. Quercetin is present in plants, and it presents many different glycosidic forms (formed by attaching a glycosyl group), which directly reflects on the bioavailability and bioactivity profile of this substance. However, a part of the absorbed quercetin can circulate in the blood as aglycone (the lacking sugar moieties form). Quercetin can act beneficially in several pathologies, especially those that affect the lungs [12-14]. A recent study by our research group showed that quercetin acts as a potent agent in preventing oxidative damage and pulmonary changes [15]. Some studies also extend the effects of quercetin to liver pathologies, exerting a modulating action on inflammation, fibrosis, and redox imbalance through the interaction with a wide spectrum of central molecular targets for cell signaling machines, thus playing a promising role in the treatment of chronic inflammatory liver diseases [16, 17]. However, the ability of quercetin to counteract the deleterious effects of cigarette smoke in the liver has not been elucidated. The goal of this study was to evaluate the effects of quercetin on reducing the redox imbalance and liver inflammation induced by long-term cigarette smoke exposure.

\section{Material and Methods}

2.1. Animals. The ethics committee of the Federal University of Ouro Preto (2015/20) approved all animal experiments, and the experiments were performed according to the rules of animal protection and the ethical principles of the Brazilian Society of the Science in Laboratory Animals (SBCAL).

Forty male C57BL/6 mice (12 weeks old) obtained from the Laboratory of Experimental Nutrition (LABNEX) at the Federal University of Ouro Preto (UFOP) were housed under controlled conditions ( $12 \mathrm{~h}$ light/dark, $21^{\circ} \mathrm{C} \pm 2^{\circ} \mathrm{C}, 50 \% \pm 10$ $\%$ humidity) with access to food and water ad libitum. The mice were divided into 5 groups ( $n=8$ per group): a control group exposed to ambient air (CG), a group that received $200 \mu \mathrm{L}$ of vehicle solution containing $50 \%$ propylene glycol (Sigma Aldrich, Missouri, USA) and 50\% saline (VG) by orogastric gavage, a group that received $10 \mathrm{mg} / \mathrm{kg} /$ day of quercetin (Sigma Aldrich, Missouri, USA) diluted in $200 \mu \mathrm{L}$ of propylene glycol solution (QG), a group exposed to cigarette smoke (CSG), and a group administered with quercetin and exposed to cigarette smoke (QCSG). The administration of quercetin was performed via orogastric gavage $1 \mathrm{~h}$ before exposure to cigarette smoke $[12,13]$.

2.2. Cigarette Smoke Exposure Protocol. In a smoking chamber, the mice were exposed to 12 commercial full-flavor filtered Virginia cigarettes (tar: $10 \mathrm{mg}$, nicotine: $0.9 \mathrm{mg}$, and carbon monoxide: $10 \mathrm{mg}$ ) per day divided into three times daily (morning, afternoon, and night) for 60 consecutive days as previously described [18]. The cigarette smoke (CS) and
QCSG animals were initially placed in an inhalation chamber $(40 \mathrm{~cm} \times 30 \mathrm{~cm} \times 25 \mathrm{~cm})$ inside an exhaustion chapel. Each cigarette was coupled to a $60 \mathrm{~mL}$ plastic syringe through which the smoke was injected inside the inhalation chamber. CSG and QCSG were maintained in the smoke atmosphere for $6 \mathrm{~min}$; then, the cover was removed from the inhalation chamber for $1 \mathrm{~min}$ to allow the air to completely exhaust. The procedure was then repeated with the remaining cigarettes. The CG, VG, and QG were subjected to the same conditions, but without exposure to cigarette smoke. At the end of the exposition, the animals were also weighed on a digital scale (Mark ${ }^{\circledR}$; Série M/Bel Analytical Equipment LTDA, São Paulo, Brazil).

2.3. Blood and Liver Collection. The animals were euthanized twenty-four hours after the last exposure to CS by an overdose of ketamine $(130 \mathrm{mg} / \mathrm{kg})$ and xylazine $(0.3 \mathrm{mg} / \mathrm{kg})$. The blood was collected through the cardiac puncture in polypropylene tubes containing $15 \mu \mathrm{L}$ of anticoagulants. Subsequently, it was centrifuged at $10.000 \mathrm{rpm}$ for 15 minutes, and the plasma was collected and stored in a freezer $\left(-80^{\circ} \mathrm{C}\right)$. Immediately after euthanasia, the right ventricle was perfused with saline to remove blood from the liver; subsequently, two aliquots of liver were separated. The first sample was fixed in $4 \%$ neutral buffered formalin, dehydrated, cleared, embedded in paraffin, cut into $4-5 \mu \mathrm{m}$ sections, and stained with hematoxylin and eosin (H\&E) for histopathological analyses. Afterwards, $100 \mathrm{mg}$ of the tissue was placed in polypropylene tubes with $1 \mathrm{~mL}$ of phosphate buffer ( $\mathrm{pH} 7.8$ ), and it was homogenized in a tissue homogenizer. Then, the samples were centrifuged at $4^{\circ} \mathrm{C}$ for 10 minutes and $13.000 \mathrm{rpm}$ (MIKRO 200R; laboratory technology Hettich, Tuttlingen, Germany), and the supernatant was collected and stored at $-80^{\circ} \mathrm{C}[15]$.

2.4. Biochemical Measurements. The samples containing $300 \mu \mathrm{L}$ of plasma were sent to the Pilot Laboratory of Clinical Analysis (Lapac) in Ouro Preto-MG, in order to determine the concentrations of liver function markers, including aspartate aminotransferase (AST; K048-6), alanine aminotransferase (ALT; K049-6), lactate dehydrogenase (LDH; K014-2,) and gamma-glutamyltransferase (GGT-1; K0802 ), which were determined by an automatic spectrophotometry in the clinical analyzer Randon Acess Clinical Analyzer, Wiener Lab, model CM-200 (WIENER LAB, Rosario, Argentina) by the enzymatic-colorimetric method using specific kits that were purchased from Bioclin ${ }^{\circledR}$, Quibasa (MG, Brazil).

2.5. Histopathological Analysis. The histopathological analysis was carried out using a light microscope (Leica DFC 300 FX) with a 40x microscopic objective. For histological and morphometric analysis, we obtained twenty randomly chosen images of histological slides that were prepared from the liver sections. These slides were scanned using the Leica Application software. Histological screening focused on the detection and grading of the following abnormalities: inflammatory reaction, sinusoidal congestion, degenerative hepatocyte lesions (hydropic and steatotic), sinusoidal congestion, 
hyperemia, bleeding focus, intralobular and portal granulomas, fibrosis, and necrosis. The changes in the experimental histopathological parameters present were semiquantified according to a scoring method as (0) absent, (1) mild, when present in approximately less than $33 \%$ in the total area of the tissue, (2) moderate, when present between approximately $33 \%$ and $66 \%$ in the total area of the tissue, and (3) intense, when present in approximately more than $66 \%$ in the total area of the tissue $[19,20]$. The counting of the number of inflammatory cells present in the hepatic lobes included the resident Kupffer cells. Slides were analyzed using the Leica Q-Win Plus software (Leica Microsystems, Inc., Buffalo Grove, Illinois, USA).

2.6. Analysis of Antioxidant Defense and Biomarkers of Oxidative Stress. A hepatic homogenate was used for the analysis of antioxidant defense and oxidative damage. The superoxide dismutase (SOD) activity was measured according to the method described by Marklund and Marklund, based on the enzyme ability to inhibit the autoxidation of pyrogallol [21]. The catalase (CAT) activity was measured using a spectrophotometer according to the method described by Aebi, which is based on the decrease in $\mathrm{H}_{2} \mathrm{O}_{2}$ at an absorbance of $240 \mathrm{~nm}$ [22]. The glutathione analysis was determined using an assay adapted from a commercial kit (CS0260, Sigma, St. Louis, MO, USA), and the method is based on the ability to reduce the $5.5^{\prime}$-Dithio Acid-bis(2-nitrobenzoic) to thio-2-nitrobenzoic acid according to the Griffith assay [23]. In order to calculate the concentration of total glutathione (GSH+GSSG) and oxidized glutathione (GSSG), a standard serial dilution curve was prepared, and the concentration of reduced form (GSH) was calculated from the subtraction of the total glutathione value by the value of oxidized glutathione. In order to measure lipid peroxidation, we used the method described by Buege and Aust [24], which thiobarbituric acid reacts with oxidized lipids and the concentration of TBARS was provided using the molar extinction coefficient, following the law of Lambert Beer. For the determination of the carbonylated protein, a protocol adapted from the methodology described by Reznick and Packer was used [25]. The total protein was measured according to the Lowry method [26].

2.7. Immunoenzymatic Assay for Inflammatory Mediators. Interleukin-6 (IL-6), interleukin-10 (IL-10), monocyte chemotactic protein 1 (CCL2), and interferon- $\gamma$ (INF- $\gamma$ ) were performed in liver homogenate by the enzyme-linked immunosorbent assay (ELISA) method. The assays were performed using commercial kits (PeproTech, Ribeirão Preto, Brazil), and the antibodies and reagents were prepared according to the instructions from the manufacturer. Immunoenzymatic assays were performed in 96-well plates, on which $100 \mu \mathrm{L}$ of monoclonal antibody was added to the protein (or peptide) of interest, and the sample were diluted in phosphatebuffered saline (PBS) containing $0.1 \%$ fetal bovine serum. After incubation for 12 hours at room temperature, the nonadsorbed antibodies were discarded, and blocking was performed with a solution containing PBS with $1 \%$ fetal bovine serum for one hour. Samples of the liver tissue supernatant, which were previously processed and stored at $-80^{\circ} \mathrm{C}$, were applied in a volume of $50 \mu \mathrm{L}$ for each well. The reading of the staining intensity was performed in an ELISA reader using a wavelength of $490 \mathrm{~nm}$. The quantification of chemokines and interleukins present in the samples was determined based on the optical density obtained with the peptide standard curve, analyzed by the SOFT Max PRO 4.0 software $[27,28]$, and the result obtained was a cytokine picogram per milliliter $(\mathrm{pg} / \mathrm{mL})$.

2.8. Statistical Analysis. The statistical analyses were performed using Prism version 5.00 for Windows 7 (GraphPad Software, San Diego, CA USA). The normal distribution of each variable was assessed using the Kolmogorov-Smirnov test. The normally distributed data were analyzed with oneway ANOVA followed by Tukey's posttest, and the data are expressed as mean \pm standard error of the mean (SEM). For nonparametric data, we used the Kruskal Wallis test followed by Dunn's posttest, and the data are expressed as the median, minimum, and maximum values. In both cases, the difference was considered significant when the $p$ value was $<0.05$.

\section{Results}

3.1. Hepatic Function Markers and Body Weight. The animals from the five experimental groups were evaluated to investigate the effects of cigarette smoke on liver damage biomarkers. It can be seen from Table 1 that a higher level of ALT (ANOVA, $p=0.0071$ ) in the animals was exposed to long-term cigarette smoke (CSG) when compared with the control groups $(p<0.05)$. However, there were no significant differences between the CSG and QCSG regarding the markers of the liver function (Table 1).

There was a decrease of the final body weight (ANOVA, $p<0.0001)$ of the CSG compared to CG and VG $(p=0.001)$. However, there was no difference with respect to the final body weight between CSG and QCSG $(p>0.05)$ (Table 1).

3.2. Histopathological Aspects. The semiquantitative analyzes were performed for the presence and intensity of hepatic lesions of mice from the experimental groups. The histopathological evaluation revealed that the control group (CG) had almost completely preserved liver histology (Figure 1(a), Tables 2 and 3). Exposure to cigarette smoke (CSG) resulted in changes in the liver parenchyma such as inflammatory reaction, sinusoidal congestion, hyperemia, granulomatous reaction, and bleeding focus (Figures 1(b)-(e), Tables 2 and 3). The quercetin administration (QCSG) was able to improve the effects of long-term cigarette smoke exposure, with decreasing or the disappearing of injuries (Figure 1(f), Tables 2 and 3). Hydropic degeneration was observed in all groups; however, there were no significant differences (Figure 1, Tables 2 and 3).

The semiquantitative analysis showed higher frequency of sinusoidal congestion/hyperemia in livers of mice exposed to long-term cigarette smoke (75\%) than all groups (CG-0\%, VG-12.5\%, QG-12.5\%, QCSG-12.5\%) (Table 2). Moreover, the quercetin administration associated to CS (QCSG) provoked an increase in the severity of this injury $(12.5 \%)$ 
TABLE 1: Serum biochemical parameters and body weight of C57BL/6 mice.

\begin{tabular}{lccccc}
\hline & CG & VG & QG & CSG & QCSG \\
\hline ALT (U/L) & $12.41 \pm 1.17$ & $12.56 \pm 1.90$ & $11.56 \pm 1.72$ & $24.50 \pm 4.75^{\mathrm{a}, \mathrm{b}, \mathrm{c}}$ & $18.80 \pm 2.27$ \\
AST (U/L) & $88.36 \pm 5.45$ & $96.85 \pm 10.4$ & $88.26 \pm 5.49$ & $83.80 \pm 6.93$ & $83.80 \pm 4.40$ \\
GGT-1 (U/L) & $2.46 \pm 0.82$ & $2.81 \pm 0.61$ & $4.87 \pm 0.42$ & $3.38 \pm 0.56$ & $2.98 \pm 0.50$ \\
LDH (U/L) & $386.2 \pm 31.1$ & $412.2 \pm 23.5$ & $356.4 \pm 18.8$ & $383.5 \pm 26.6$ & $389.4 \pm 45.0$ \\
Body weight (g) & $27.78 \pm 0.38$ & $26.83 \pm 0.31$ & $25.60 \pm 0.97$ & $24.11 \pm 0.65^{\mathrm{a}, \mathrm{b}}$ & $23.47 \pm 0.34^{\mathrm{a}, \mathrm{b}}$ \\
\hline
\end{tabular}

CG: control group; VG: vehicle group; QG: quercetin group; CSG: cigarette smoke group; QCSG: quercetin and cigarette smoke group, ALT: alanine aminotransferase; AST: aspartate aminotransferase; GGT-1: gamma-glutamyltransferase; LDH: lactate dehydrogenase. ${ }^{\text {a }}$ represents a significant difference between groups when compared to CG; ${ }^{b}$ represents a significant difference between groups when compared to VG; ${ }^{c}$ represents a significant difference between groups when compared to QG. ALT, AST, GGT-1, LDH, and body weight were expressed as mean \pm standard error of the mean and were analyzed by one-way ANOVA followed by Tukey's posttest, $n=5-8$ animals per group $(p<0.05)$.

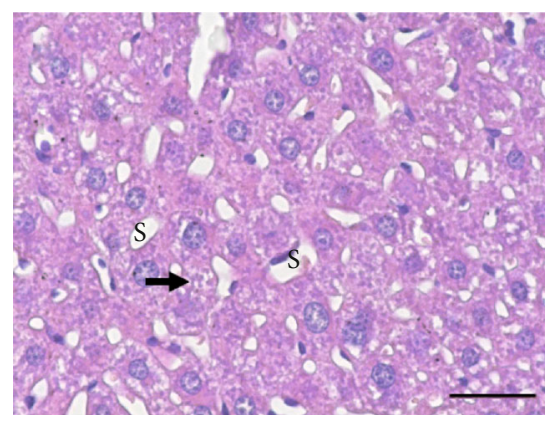

(a)

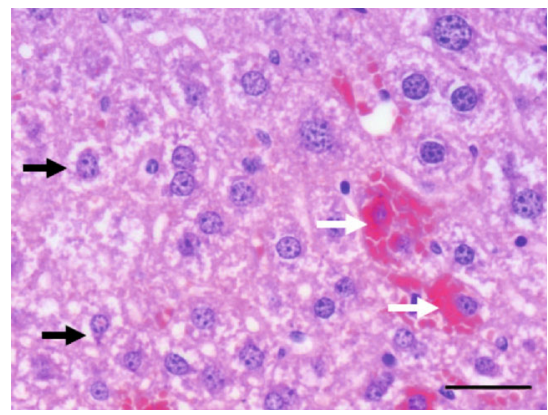

(c)

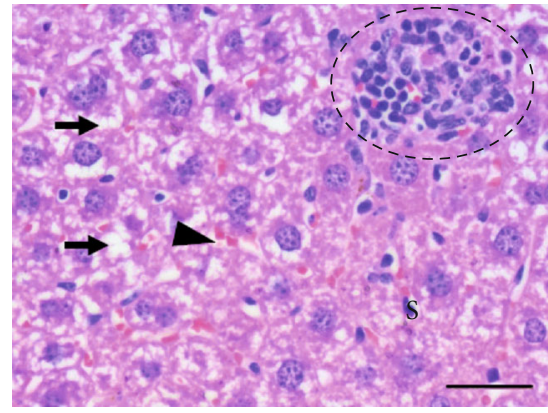

(e)

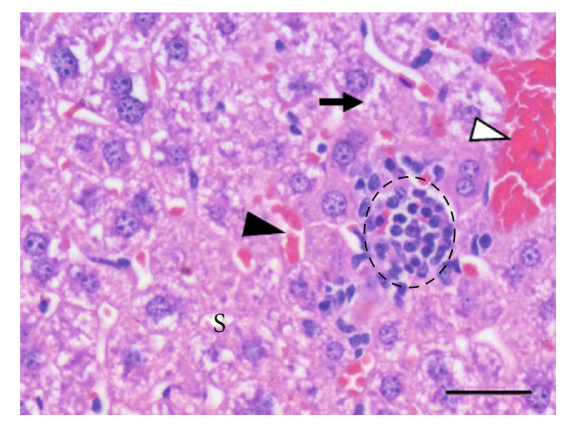

(b)

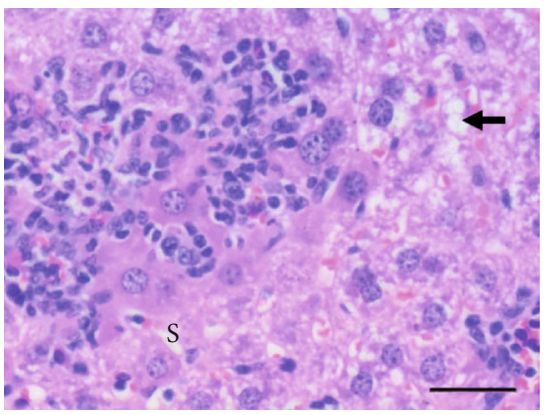

(d)

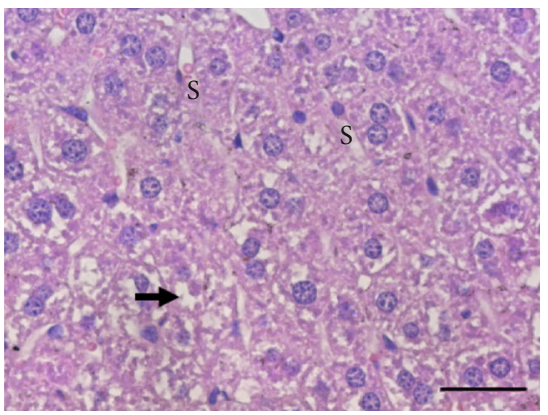

(f)

Figure 1: Histopathological aspects of the liver. Photomicrographs of liver sections stained with hematoxylin and eosin. Bar $=50 \mu \mathrm{m}, 400 \mathrm{x}$ magnification. (a) Representative image of groups exposed to ambient air (CG, VG, QG). (b)-(e) Representative images of the lesions found in the group exposed to cigarette smoke (CSG). (f) Representative image of the group pretreated with quercetin and exposed to cigarette smoke (QCSG). (a) Preserved liver parenchyma, presence of hydropic degeneration (black arrow), and preserved sinusoid capillaries (S). (b) Hydropic degeneration (black arrow), congestion of sinusoid (black arrowhead), presence of granuloma (dotted circle), and hyperplasia (white arrowhead). (c) Hydropic degeneration (black arrow) and bleeding focus (white arrow). (d) Inflammatory cell infiltration and hydropic degeneration (black arrow). (e) Hydropic degeneration (black arrow), congestion of sinusoid (black arrowhead), and presence of granuloma (dotted circle). (f) Preserved hepatic parenchyma notes the hydropic degeneration (black arrow) and well-preserved sinusoid capillaries (S). 
TABLE 2: Frequency of histopathological changes in the liver of C57BL/6 mice in the experimental groups.

\begin{tabular}{|c|c|c|c|c|c|}
\hline Lesions & $\begin{array}{c}\mathrm{CG} \\
N=8(\%)\end{array}$ & $\begin{array}{c}\mathrm{VG} \\
N=8(\%)\end{array}$ & $\begin{array}{c}\text { QG } \\
N=8(\%)\end{array}$ & $\begin{array}{c}\text { CSG } \\
N=8(\%)\end{array}$ & $\begin{array}{c}\text { QCSG } \\
N=8(\%)\end{array}$ \\
\hline Hydropic degeneration & $8(100)$ & $8(100)$ & $8(100)$ & $8(100)$ & $8(100)$ \\
\hline Sinusoidal congestion/hyperemia & $0(0.0)$ & $1(12.5)$ & $1(12.5)$ & $6(75.0)$ & $1(12.5)$ \\
\hline Bleeding focus & $0(0.0)$ & $0(0.0)$ & $0(0.0)$ & $2(25.0)$ & $0(0.0)$ \\
\hline Granulomas & $0(0.0)$ & $0(0.0)$ & $0(0.0)$ & $6(75.0)$ & $0(0.0)$ \\
\hline Inflammatory reaction & $0(0.0)$ & $2(25.0)$ & $1(12.5)$ & $7(87.5)$ & $1(12.5)$ \\
\hline Fibrosis & $0(0.0)$ & $0(0.0)$ & $0(0.0)$ & $0(0.0)$ & $0(0.0)$ \\
\hline Necrosis & $0(0.0)$ & $0(0.0)$ & $0(0.0)$ & $0(0.0)$ & $0(0.0)$ \\
\hline
\end{tabular}

CG: control group; VG: vehicle group; QG: quercetin group; CSG: cigarette smoke group; QCSG: quercetin and cigarette smoke group.

TABLE 3: The semiquantitative score values (score minimum-score maximum) of the main histological lesions found in the livers of experimental animals.

\begin{tabular}{lccccc}
\hline Lesions & CG & VG & QG & CSG & QCSG \\
\hline Hydropic degeneration & $1(1-2)$ & $1(1-2)$ & $1(1-2)$ & $3(1-3)$ & $1(1-3)$ \\
Sinusoidal congestion/hyperemia & 0 & $0(0-1)$ & $0(0-1)$ & $2(0-3)^{\mathrm{abc}}$ & $0(0-1)^{\mathrm{d}}$ \\
Inflammatory reaction & 0 & $0(0-1)$ & $0(0-1)$ & $1(0-3)^{\mathrm{abc}}$ & $0(0-1)^{\mathrm{d}}$ \\
\hline
\end{tabular}

CG: control group; VG: vehicle group; QG: quercetin group; CSG: cigarette smoke group; QCSG: quercetin and cigarette smoke group. ${ }^{\mathrm{a}}$ represents a significant difference between CSG when compared to CG; ${ }^{b}$ represents a significant difference between CSG when compared to VG; ${ }^{\mathrm{c}}$ represents a significant difference between CSG when compared to QG; ${ }^{\mathrm{d}}$ represents a significant difference between groups when compared to CSG. Sinusoidal congestion/hyperemia $(p=0.0008)$. Inflammation reaction $(p=0.0003)$. The data were analyzed by Kruskal-Wallis followed by Dunn's posttest $(n=8$ animals per group).

compared to CSG $(p=0.0008)$ (Table 3$)$. In the CSG, this lesion ranged from absent $(25 \%)$, moderate (25\%), to intense $(50 \%)$ in severity (Table 3 ). While in the other groups, the sinusoidal congestion/hyperemia ranged only from absent (CG: $100 \%$, VG: $87.5 \%$, QG: $87.5 \%$, QCSG: $87.5 \%$ ) to mild intensity (VG: 12.5\%, QG: 12.5\%, QCSG: 12.5\%) (Table 3).

The bleeding focus and granulomatous reactions were observed only in animals exposed to long-term cigarette smoke (CSG) (25\% and 75\%, respectively) (Figures 1(b), $1(\mathrm{c})$, and 1(e) and Table 2).

The inflammatory reaction was more frequent in the group exposed to CS (CSG) (87.5\%) than VG (25\%) and QG (12.5\%) (Figures 1(b), 1(d), and 1(e) and Table 2). There was not any inflammatory reaction in the control group (CG: $0 \%$ ); in these animals, only the resident inflammatory cells were observed (Figure 1(a), Table 2). The quercetin administration associated to cigarette smoke (QCSG) decreased the frequency of the inflammatory reaction to $12.5 \%$ of the animals $(p=0.0003)$ (Table 2), i.e., the inflammatory reaction reduced to values similar to those of the VG and QG. Moreover, the inflammatory reaction was more intense in CSG, presenting a mild range from (37.5\%) to moderate (37.5\%) to intense, while the other groups presented only mild intensity, when observed (VG: 25\%, QG: 12.5\%, QCSG: 12.5\%) (Table 3).

Moreover, hepatic fibrosis and necrosis were not observed in the animals studied (Table 2).

The number of inflammatory cells to the liver was greater (ANOVA, $p<0.0001$ ) in animals exposed to cigarette smoke (CSG) $(29.43 \pm 2.24)$ compared to control groups (CG: $16.51 \pm 0.11$, VG: $18.39 \pm 0.41$, QG: $16.83 \pm 0.49)(p=0.001$ ). Pretreatment with quercetin $(14.82 \pm 0.52)$ resulted in a lower number of inflammatory cells in the liver when com-

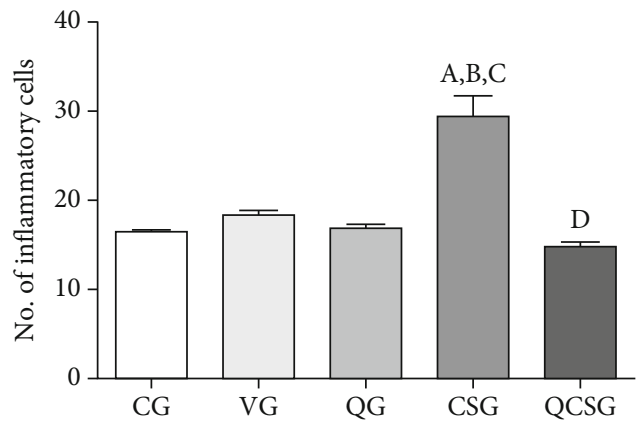

FIGURE 2: Number of inflammatory nucleus into the hepatic parenchyma of C57BL/6 mice. CG: control group; VG: vehicle group; QG: quercetin group; CSG: cigarette smoke group; QCSG: quercetin and cigarette smoke group. ${ }^{a}$ represents a significant difference between groups when compared to CG. ${ }^{b}$ represents a significant difference between groups when compared to VG. ${ }^{c}$ represents a significant difference between groups when compared to QG. $\mathrm{d}$ represents a significant difference between groups when compared to CSG. Data were expressed as mean \pm standard error of the mean and were analyzed by one-way ANOVA followed by Tukey's posttest, $n=8$ animals per group $(p<0.05)$.

pared to CSG $(p=0.001)$ and presented values similar to those of the control group (Figure 2).

3.3. Antioxidant Defense and Oxidative Stress Biomarkers in the Liver. Cigarette smoke exposure promoted an increase of the SOD activity (ANOVA, $p=0.0009$ ) compared to CG, VG, and PG $(p<0.05)$, which was attenuated by pretreatment with quercetin $(p=0.001)$. The catalase activity was lower (ANOVA, $p=0.0019$ ) in the CSG and QCSG groups 
TABLE 4: Antioxidant defense and oxidative stress biomarkers in liver homogenate.

\begin{tabular}{lccccc}
\hline & CG & VG & QG & CSG & QCSG \\
\hline SOD (U/mg protein) & $90.74 \pm 4.72$ & $88.01 \pm 5.0$ & $93.26 \pm 5.42$ & $115.4 \pm 5.85^{\mathrm{a}, \mathrm{b}, \mathrm{c}}$ & $81.42 \pm 5.36^{\mathrm{d}}$ \\
CAT (U/mg protein) & $0.28 \pm 0.02$ & $0.20 \pm 0.03$ & $0.22 \pm 0.01$ & $0.13 \pm 0.03^{\mathrm{a}}$ & $0.14 \pm 0.02^{\mathrm{a}}$ \\
GSH/GSSG ratio & $7.21(2.59 ; 13.30)$ & $5.84(4.11 ; 6.85)$ & $6.05(5.24 ; 9.04)$ & $0.41(0.34 ; 1.40)^{\mathrm{c}}$ & $6.61(3.19 ; 10.64)^{\mathrm{d}}$ \\
TBARS (nmol/mg protein) & $0.34 \pm 0.02$ & $0.24 \pm 0.02$ & $0.31 \pm 0.02$ & $2.07 \pm 0.50^{\mathrm{a}, \mathrm{b}, \mathrm{c}}$ & $0.27 \pm 0.04^{\mathrm{d}}$ \\
Protein carbonyl (nmol/ng protein) & $12.73 \pm 1.28$ & $13.13 \pm 1.90$ & $16.07 \pm 2.05$ & $16.18 \pm 0.93$ & $14.12 \pm 1.58$ \\
\hline
\end{tabular}

CG: control group; VG: vehicle group; QG: quercetin group; CSG: cigarette smoke group; QCSG: quercetin and cigarette smoke group, SOD: superoxide dismutase; CAT: catalase; GSH: glutathione sulfide; GSSG: oxidized glutathione; TBARS: thiobarbituric acid reactive substances. ${ }^{a}$ represents a significant difference between groups when compared to CG; ${ }^{b}$ represents a significant difference between groups when compared to VG; ${ }^{c}$ represents a significant difference between groups when compared to QG; ${ }^{\mathrm{d}}$ represents a significant difference between groups when compared to CSG. SOD, CAT, TBARS, and protein carbonyl were expressed as mean \pm standard error of the mean and were analyzed by one-way ANOVA followed by Tukey's posttest, $n=5-8$ animals per group $(p<0.05)$. The GSH/GSSG ratio data were expressed in median, minimum, and maximum value and were analyzed by Kruskal-Wallis followed by Dunn's posttest that was used, $n=5-8$ animals per group $(p<0.05)$.

TABLE 5: Inflammatory markers in the liver of experimental groups.

\begin{tabular}{lccccc}
\hline & CG & VG & QG & CSG & QCSG \\
\hline CCL2 $(\mathrm{pg} / \mathrm{mL})$ & $4025.3 \pm 137.6$ & $3279.4 \pm 250.9$ & $3153.2 \pm 134.4$ & $1852.4 \pm 303.7^{\mathrm{a}, \mathrm{b}, \mathrm{c}}$ & $3693.6 \pm 175.9^{\mathrm{d}}$ \\
INF- $\gamma(\mathrm{pg} / \mathrm{mL})$ & $537.56 \pm 11.85$ & $513.25 \pm 42.14$ & $559.05 \pm 42.42$ & $324.62 \pm 21.59^{\mathrm{a}, \mathrm{b}, \mathrm{c}}$ & $519.28 \pm 24.11^{\mathrm{d}}$ \\
IL-10 $(\mathrm{pg} / \mathrm{mL})$ & $1466.3 \pm 99.79$ & $1529.2 \pm 149.5$ & $1471.6 \pm 43.6$ & $2342.3 \pm 218.1^{\mathrm{a}, \mathrm{b}, \mathrm{c}}$ & $1698.6 \pm 57.5^{\mathrm{d}}$ \\
IL-6 $(\mathrm{pg} / \mathrm{mL})$ & $1711.2 \pm 57.7$ & $1712.0 \pm 49.6$ & $1688.1 \pm 48.1$ & $1780.6 \pm 57.3$ & $1668.7 \pm 56.1$ \\
\hline
\end{tabular}

CG: control group; VG: vehicle group; QG: quercetin group; CSG: cigarette smoke group; QCSG: quercetin and cigarette smoke group, CCL2 or MCP1: monocyte chemotactic protein 1 ; INF- $\gamma$ : interferon- $\gamma$; IL-10: interleukin 10; IL-6: interleukin $6 .{ }^{a}$ represents a significant difference between groups when compared to CG; ${ }^{\mathrm{b}}$ represents a significant difference between groups when compared to VG; ${ }^{\mathrm{c}}$ represents a significant difference between groups when compared to QG; ${ }^{d}$ represents a significant difference between groups when compared to CSG. Data were expressed as mean \pm standard error of the mean and were analyzed by one-way ANOVA followed by Tukey's posttest, $n=8$ animals per group $(p<0.05)$.

compared to the control $(p=0.01)$. The GSH/GSSG ratio was lower (Kruskal Wallis, $p=0.0158$ ) in the CSG than QC $(p<0.05)$, and the administration of quercetin increased the GSH/GSSG ratio compared to CSG $(p<0.05)$. The damage to liver parenchyma was evaluated based on levels of TBARS (ANOVA, $p<0.0001$ ); as a result, the lipid oxidation was higher in the group exposed to cigarette smoke compared to CG, VG, and QG $(p=0.001)$, and the lipid oxidation was also attenuated by pretreatment with quercetin $(p=0.001)$ (Table 4).

3.4. Inflammatory Mediators' Levels in the Liver. Inflammatory markers IL-6, IL-10, INF- $\gamma$, and CCL2 were measured in order to assess the inflammatory status of the liver. The animals exposed to cigarette smoke for sixty days showed higher levels of INF- $\gamma$ (ANOVA, $p=0.0002$ ) and IL-10 (ANOVA, $p=0.0005$ ) in liver homogenate when compared with CG, VG, and QG $(p=0.01)$. For both markers, pretreatment with quercetin led to a decrease in cytokine levels compared to CSG $(p<0.05)$. The levels of CCL2 chemokine (ANOVA, $p<0.0001$ ) in the liver of the group exposed to smoke were lower than the control group $(p=0.001)$, and the administration of quercetin promoted an increase in the levels of this marker compared to CSG $(p=0.001)$. For the analysis of IL-6, no statistical difference was observed between the experimental groups (ANOVA, $p=0.65$ ) (Table 5).

\section{Discussion}

In this study, we investigated the antioxidant effect of quercetin against the oxidative stress, inflammation, and changes in liver morphology induced by cigarette smoke. We observed morphological changes and redox imbalance in the livers of mice exposed to long term-cigarette smoke, strengthening the deleterious effect of cigarette smoke on the liver parenchyma, corroborating previous studies [29-31]. A study conducted by Ogenyi et al. revealed an adverse effect of passive cigarette smoke on the liver morphology and biochemistry of Wistar rats [31]. Moreover, a study conducted by Adedayo et al. reported that the smoke extract of tobacco nicotine has adverse and compromising effects on the liver of male Sprague-Dawley rats [30].

Liver diseases represent an important focus of attention in view of the multiple functions of the liver in the body. Use of herbal drugs in the treatment of liver diseases has a long tradition [17]. In this context, quercetin, the most abundant flavonoid in nature, has been found to exhibit a potent antioxidant capacity and the ability to counteract several insults to the liver. Kalantari et al. demonstrated that quercetin is effective for the prevention of tert-butyl hydroperoxideinduced hepatic damage in mice [32]. Similarly, Lee et al. reported that quercetin prevents liver inflammatory injury, induced in rats by ethanol extracts from tartary buckwheat [33]. However, to our knowledge, this is the first study that 
examines the antioxidant effect of quercetin against deleterious changes in liver morphology and in the oxidative stress induced by cigarette smoke.

We observed that there was a higher level of ALT in the animals exposed to long-term cigarette smoke when compared with the control group. ALT is one of the most used biomarkers for measuring liver injury, and several studies with cigarette smoke use this biomarker to estimate the harmful effects of smoke on the liver tissue $[9,34,35]$. Studies describe the role of quercetin in reducing liver damage, in face of an injury-causing agent, by determining ALT levels [36-38]. However, despite the findings of the current study showing that quercetin combats ROS, inflammation, and helps with antioxidant defense, there was no statistical difference between the ALT levels between CSG and QCSG.

Previous studies have demonstrated a reduction in the body weight in animals exposed to cigarette smoke $[1,18$, 39]. A study by our research group correlated this reduction in the body weight with inflammation and subsequent secretion of TNF- $\alpha$, in addition to food satiety in animals exposed to cigarette smoke [18]. Here, our results corroborate with these studies, where a decrease in the body weight was observed in the animals exposed to CS, perhaps as a consequence of the inflammatory process in the liver tissue. Pretreatment with quercetin was not able to reverse this parameter. Our results are in agreement with other studies that did not observe changes in the body weight with low concentration of quercetin [40-42].

We observed that the quercetin administration was able to improve the effects of long-term cigarette smoke exposure, with injury decreases resulting from this exposure, such as inflammatory reaction, sinusoidal congestion, hyperemia, granulomatous reaction, and bleeding focus. Moreover, we observed a significant increase in the number of inflammatory cells into the liver in response to cigarette smoke exposure that may be directly related to the time of exposure to cigarette smoke, which was long. Several studies showed a relationship between cigarette smoke and a greater influx of total leukocytes, either by the formation of reactive oxygen species or by the presence of nicotine [43-45]. The quercetin reduced the number of inflammatory cells to the liver in animals exposed to long-term cigarette smoke, supporting the cytoprotective and anti-inflammatory effects of quercetin [46]. A similar result showed by Barros et al. found evidence of the hepatoprotective quercetin activity in acute paracetamol toxicity, as shown by a reduction in necrosis and inflammation [47].

Previous data showed that the cigarette smoke exposure promoted changes in the antioxidant enzyme activity and oxidative damage in the liver of mice and rats $[48,49]$. We evaluated the redox imbalance by measuring oxidative damage, and our results showed that the cigarette smoke significantly increased the activity of the SOD, and the quercetin administration restored their activity to control levels. These results prove the ability of quercetin to combat and neutralize reactive oxygen species (ROS), preventing oxidative stress according to Dias et al. [50]. These authors found that quercetin, by scavenging ROS, prevents the elevation of the SOD activity in the liver of streptozotocin-induced diabetic rats.
The increase in the SOD activity in the liver tissue caused by cigarette smoke has also been described $[51,52]$ in rats.

GSH is a key player in the maintenance of the cellular redox status and exists primarily in two redox forms, reduced (GSH) and oxidized (GSSG) [53]. The GSH/GSSG ratio can serve as a good indicator of the cellular redox state [54]. Our results indicated that CS reduced the GSH/GSSG ratio, while quercetin, in turn, was able to restore the GSH/GSSG ratio. This result possibly indicates a greater activity of GPx in the group exposed to cigarette smoke and a lower activity of this enzyme in group pretreated with quercetin and exposed to cigarette smoke. Therefore, we believe that quercetin will neutralize ROS instead of GPx, thus maintaining the redox status of the cells. Our data corroborate with the results from a previous study by Olayinka et al. and Chen, 2010, which reported protective effects of quercetin, regarding the ability of quercetin to increase the GSH/GSSG ratio in liver injury induced by procarbazine and ethanol, respectively $[55,56]$. Therefore, an increase in the antioxidant system and consequent reduction of ROS induced by the quercetin administration observed in the present study may be a main factor for protection of the liver against injury caused by CS exposure.

In our experimental model, the CAT activity decreased in CSG compared to that in control groups, and the quercetin administration was unable to regulate the activity of this enzyme. Similarly, a series of studies in the lung and liver reported a decrease of the catalase activity in models that promote an increase in ROS [18, 57-59]. A depletion of catalase reserves promoted by a persistent increase in production of reactive oxygen species may have occurred. In the other hand, the liver has one of the highest organ contents of GSH $[60,61]$. The glutathione system is expected to be more active in neutralizing ROS production after exposure to cigarette smoke when compared to other components of the antioxidant system, such as the CAT activity. Despite the decreased CAT activity, quercetin showed a strong antioxidant activity by maintaining oxidative balance. According to $\mathrm{Xu}$ et al., the antioxidant activity of quercetin is mainly manifested through its effect on glutathione [62].

In this study, the long-term cigarette smoke significantly increased the TBARS levels that were restored by the quercetin administration. These results are in accordance with previous studies that evaluated the effect of cigarette smoke on the liver $[34,52,63]$. Some studies in liver damage have also demonstrated that quercetin modulates lipid and lipoprotein metabolism damage caused by oxidative stress inducing agents other than cigarette smoke [32]. Our results demonstrate that quercetin is effective in decreasing lipid peroxidation and in preventing oxidative damage in the liver parenchyma of mice exposed to long-term cigarette smoke.

We observed a decrease of CCL2 and IFN- $\gamma$, as well as an increase of IL-10 in the C57BL/6 mice exposed to long-term CS. Regarding the levels of IL- 6 in the hepatic homogenate, there were no changes observed between the experimental groups. As the primary injury of cigarette smoke occurs in the lung, it is expected that these inflammatory markers are more active in this organ, compared to other organs such as the liver [18]. The IL-10 increase in the liver tissue of mice 
exposed to CS may be a response to the influx of inflammatory cells to that organ, and the concomitant decrease in proinflammatory mediators, such as CCL2 and INF- $\gamma$, may be a consequence of the IL-10 which regulates the proinflammatory activity of these mediators, and it has been described in immunologic studies in several organs $[44,64,65]$. Studies describe the regulation of IL-10 over other cytokines and chemokines, and, depending on the inflammatory profile of the region with injury caused by oxidant agents, it exerts a modulation on INF- $\gamma$ and CCL2 $[66,67]$.

Our data demonstrated that the pretreatment with quercetin restored the levels of inflammatory markers to the levels of the control group and are in agreement with those of Chen, who demonstrated the role of quercetin in restoring cytokine and chemokine levels in the liver tissue exposed to ethanol [56]. Other works have also described in several organs, the role of quercetin in modulating inflammatory response through the induction or repression of pro or anti-inflammatory cytokines [13, 18, 33, 46, 68]. However, this is the first study that examines the antioxidative and anti-inflammatory properties of quercetin against deleterious changes in liver morphology and in the oxidative damage induced by cigarette smoke.

In summary, these results suggest that quercetin has a promising role as an auxiliary pharmacological tool in the treatment of damage to the liver due to the exposure to cigarette smoke.

\section{Data Availability}

I declare that data related to the manuscript entitled "Protective effects of quercetin on livers from mice exposed to longterm cigarette smoke" are available for publicy.

\section{Conflicts of Interest}

The author(s) declared no potential conflicts of interest.

\section{Authors' Contributions}

Pedro A. Machado-Junior and Natália P. S. Araújo contributed equally to this work.

\section{Acknowledgments}

The authors would like to express their gratitude to the Pilot Laboratory of Clinical Analysis (Lapac) for determining the concentrations of liver function markers and to Dr. Rosa Maria Esteves Arantes, from the Federal University of Minas Gerais. This work was supported by the Coordenação de Aperfeiçoamento de Pessoal de Ensino Superior (CAPES) (PNPD/CAPES-Process \# 88882.316933/2019-01 and CAPES-PVEX-Process \# 88881.172437/2018-01), Federal University of Ouro Preto (UFOP), and Conselho Nacional de Desenvolvimento Científico e Tecnológico (CNPq).

\section{References}

[1] S. Park, J. W. Kim, H. Yun et al., "Mainstream cigarette smoke accelerates the progression of nonalcoholic steatohepatitis by modulating Kupffer cell-mediated hepatocellular apoptosis in adolescent mice," Toxicology Letters, vol. 256, pp. 53-63, 2016.

[2] D. Zong, X. Liu, J. Li, R. Ouyang, and P. Chen, "The role of cigarette smoke-induced epigenetic alterations in inflammation," Epigenetics \& Chromatin, vol. 12, no. 1, article 65, 2019.

[3] G. Z. Wang, X. Cheng, X. C. Li et al., "Tobacco smoke induces production of chemokine CCL20 to promote lung cancer," Cancer Letters, vol. 363, no. 1, pp. 60-70, 2015.

[4] J. W. Westra, W. K. Schlage, A. Hengstermann et al., "A modular cell-type focused inflammatory process network model for non-diseased pulmonary tissue," Bioinformatics and Biology Insights, vol. 7, pp. 167-192, 2013.

[5] A. Dalrymple, P. Ordonez, D. Thorne, D. Dillon, and C. Meredith, "An improved method for the isolation of rat alveolar type II lung cells: use in the comet assay to determine DNA damage induced by cigarette smoke," Regulatory Toxicology and Pharmacology, vol. 72, no. 1, pp. 141-149, 2015.

[6] K. B. F. Barbosa, N. M. B. Costa, R. d. C. G. Alfenas, S. O. De Paula, V. P. R. Minim, and J. Bressan, "Oxidative stress: concept, implications and modulating factors," Revista de Nutrição, vol. 23, no. 4, pp. 629-643, 2010.

[7] C. Vaamonde-Garcia, R. R. Riveiro-Naveira, M. N. ValcarcelAres, L. Hermida-Carballo, F. J. Blanco, and M. J. LopezArmada, "Mitochondrial dysfunction increases inflammatory responsiveness to cytokines in normal human chondrocytes," Arthritis and Rheumatism, vol. 64, no. 9, pp. 2927-2936, 2012.

[8] R. F. Hoffmann, S. Zarrintan, S. M. Brandenburg et al., "Prolonged cigarette smoke exposure alters mitochondrial structure and function in airway epithelial cells," Respiratory Research, vol. 14, no. 1, p. 97, 2013.

[9] C. S. Wang, S. T. Wang, T. T. Chang, W. J. Yao, and P. Chou, "Smoking and alanine aminotransferase levels in hepatitis $\mathrm{C}$ virus infection: implications for prevention of hepatitis $C$ virus progression," Archives of Internal Medicine, vol. 162, no. 7, pp. 811-815, 2002.

[10] A. T. Nguyen-Lefebvre and A. Horuzsko, "Kupffer cell metabolism and function," Journal of enzymology and metabolism, vol., vol. 1, 2015.

[11] J. Altamirano and R. Bataller, "Cigarette smoking and chronic liver diseases," Gut, vol. 59, no. 9, pp. 1159-1162, 2010.

[12] S. Ganesan, A. N. Faris, A. T. Comstock et al., "Quercetin prevents progression of disease in elastase/LPS-exposed mice by negatively regulating MMP expression," Respiratory Research, vol. 11, no. 1, article 131, 2010.

[13] T. Yang, F. Luo, Y. Shen et al., "Quercetin attenuates airway inflammation and mucus production induced by cigarette smoke in rats," International Immunopharmacology, vol. 13, no. 1, pp. 73-81, 2012.

[14] A. Mitani, A. Azam, C. Vuppusetty, K. Ito, N. Mercado, and P. J. Barnes, "Quercetin restores corticosteroid sensitivity in cells from patients with chronic obstructive pulmonary disease," Experimental Lung Research, vol. 43, no. 9-10, pp. 417-425, 2017.

[15] N. P. da Silva Araújo, N. A. de Matos, S. L. A. Mota et al., "Quercetin attenuates acute lung injury caused by cigarette smoke both in vitro and in vivo," COPD, vol. 17 , no. 2 , pp. 205-214, 2020.

[16] A. Pavanato, M. J. Tuñón, S. Sánchez-Campos et al., "Effects of quercetin on liver damage in rats with carbon tetrachlorideinduced cirrhosis," Digestive Diseases and Sciences, vol. 48, no. 4, pp. 824-829, 2003. 
[17] R. Kabirifar, Z. A. Ghoreshi, F. Safari, A. Karimollah, A. Moradi, and E. Eskandari-Nasab, "Quercetin protects liver injury induced by bile duct ligation via attenuation of Racl and NADPH oxidase 1 expression in rats," Hepatobiliary \& Pancreatic Diseases International, vol. 16, no. 1, pp. 88-95, 2017.

[18] K. K. D. Campos, C. de Oliveira Ramos, T. L. Martins et al., "Lycopene mitigates pulmonary emphysema induced by cigarette smoke in a murine model," The Journal of Nutritional Biochemistry, vol. 65, pp. 93-100, 2019.

[19] U. G. M. de Castro, M. E. Silva, W. G. de Lima, M. J. Campagnole-Santos, and A. C. Alzamora, "Age-dependent effect of high-fructose and high-fat diets on lipid metabolism and lipid accumulation in liver and kidney of rats," Lipids in Health and Disease, vol. 12, no. 1, p. 136, 2013.

[20] G. Aydin, A. Gökçimen, M. Öncü, E. Çicek, N. Karahan, and O. Gökalp, "Histopathologic changes in liver and renal tissues induced by different doses of diclofenac sodium in rats," Turkish Journal of Veterinary and Animal Sciences, vol. 27, pp. 1131-1140, 2003.

[21] S. Marklund and G. Marklund, "Involvement of the superoxide anion radical in the autoxidation of pyrogallol and a convenient assay for superoxide dismutase," European Journal of Biochemistry, vol. 47, no. 3, pp. 469-474, 1974.

[22] H. Aebi, "[13] Catalase in vitro," Methods in Enzymology, vol. 105, pp. 121-126, 1984.

[23] O. W. Griffith, "Determination of glutathione and glutathione disulfide using glutathione reductase and 2-vinylpyridine," Analytical Biochemistry, vol. 106, no. 1, pp. 207-212, 1980.

[24] J. A. Buege and S. D. Aust, "[30] Microsomal lipid peroxidation," Methods in Enzymology, vol. 52, pp. 302-310, 1978.

[25] A. Z. Reznick and L. Packer, "[38] Oxidative damage to proteins: spectrophotometric method for carbonyl assay," Methods in Enzymology, vol. 233, pp. 357-363, 1994.

[26] O. H. Lowry, N. J. Rosebrough, A. L. Farr, and R. J. Randall, "Protein measurement with the Folin phenol reagent," The Journal of Biological Chemistry, vol. 193, no. 1, pp. 265-275, 1951.

[27] S. D. P. Fróes, A. B. F. Souza, N. A. Matos et al., "Intranasal instillation of distilled water, hypertonic saline and sodium bicarbonate promotes redox imbalance and acute lung inflammation in adult mice," Respiratory Physiology \& Neurobiology, vol. 266, pp. 27-32, 2019.

[28] E. T. Kozima, A. B. F. Souza, T. F. Castro et al., "Aluminum hydroxide nebulization-induced redox imbalance and acute lung inflammation in mice," Experimental Lung Research, vol. 46, no. 3-4, pp. 64-74, 2020.

[29] L. Azzalini, E. Ferrer, L. N. Ramalho et al., "Cigarette smoking exacerbates nonalcoholic fatty liver disease in obese rats," Hepatology, vol. 51, no. 5, pp. 1567-1576, 2010.

[30] A. D. Adedayo, A. A. Musa, A. A. Tijani, and T. D. Adeniyi, "Histological study of smoke extract of tobacco nicotiana on the heart, liver, lungs, kidney, and testes of male SpragueDawley rats," Nigerian Medical Journal, vol. 52, no. 4, pp. 217-222, 2011.

[31] S. I. Ogenyi, T. Choji, A. Chimezirim et al., "Histological and biochemical effects of cigarette smoke on the liver of Wistar rats," Annual Research \& Review in Biology, vol. 7, no. 2, pp. 119-125, 2015.

[32] H. Kalantari, H. Foruozandeh, M. J. Khodayar, A. Siahpoosh, N. Saki, and P. Kheradmand, "Antioxidant and hepatoprotec- tive effects of Capparis spinosa L. fractions and quercetin on tert-butyl hydroperoxide- induced acute liver damage in mice," Journal of Traditional and Complementary Medicine, vol. 8, no. 1, pp. 120-127, 2018.

[33] C. C. Lee, S. R. Shen, Y. J. Lai, and S. C. Wu, "Rutin and quercetin, bioactive compounds from tartary buckwheat, prevent liver inflammatory injury," Food \& Function, vol. 4, no. 5, pp. 794-802, 2013.

[34] A. Nemmar, H. Raza, D. Subramaniyan et al., "Short-term systemic effects of nose-only cigarette smoke exposure in mice: role of oxidative stress," Cellular Physiology and Biochemistry, vol. 31, no. 1, pp. 15-24, 2013.

[35] K. S. Alsalhen and R. D. Abdalsalam, "Effect of cigarette smoking on liver functions: a comparative study conducted among smokers and non-smokers male in El-Beida City, Libya," International Current Pharmaceutical Journal, vol. 3, no. 7, pp. 291-295, 2014.

[36] N. P. Sangai and R. J. Verma, "Quercetin ameliorates bisphenol A-induced toxicity in mice," Acta Poloniae Pharmaceutica, vol. 69, no. 3, pp. 557-563, 2012.

[37] K. Selvakumar, S. Bavithra, S. Suganya, F. A. Bhat, G. Krishnamoorthy, and J. Arunakaran, "Effect of quercetin on haematobiochemical and histological changes in the liver of polychlorined biphenyls-induced adult male Wistar rats," Journal of Biomarkers, vol. 2013, Article ID 960125, 2013.

[38] A. A. El-Nekeety, S. H. Abdel-Azeim, A. M. Hassan, N. S. Hassan, S. E. Aly, and M. A. Abdel-Wahhab, "Quercetin inhibits the cytotoxicity and oxidative stress in liver of rats fed aflatoxin-contaminated diet," Toxicology Reports, vol. 1, pp. 319-329, 2014.

[39] M. F. Diniz, V. A. Dourado, M. E. Silva, M. L. Pedrosa, F. S. Bezerra, and W. G. Lima, "Cigarette smoke causes changes in liver and spleen of mice newborn exposed during pregnancy," Journal of Cytology \& Histology, vol. 4, no. 1, 2013.

[40] K. Azuma, K. Ippoushi, and J. Terao, "Evaluation of tolerable levels of dietary quercetin for exerting its antioxidative effect in high cholesterol-fed rats," Food and Chemical Toxicology, vol. 48, no. 4, pp. 1117-1122, 2010.

[41] C.-S. Kim and R. Yu, "The inhibitory effect of quercetin on adipose tissue inflammation in mice fed on a high-fat diet," The Korean Journal of Obesity, vol. 23, no. 3, pp. 170-178, 2014.

[42] W. Gao, L. Pu, M. Chen et al., "Glutathione homeostasis is significantly altered by quercetin via the Keap1/Nrf2 and MAPK signaling pathways in rats," Journal of Clinical Biochemistry and Nutrition, vol. 62, no. 1, pp. 56-62, 2018.

[43] D. Nowak, U. Ruta, and G. Piasecka, "Nicotine increases human polymorphonuclear leukocytes chemotactic response-a possible additional mechanism of lung injury in cigarette smokers," Experimental Pathology, vol. 39, no. 1, pp. 37-43, 1990.

[44] D. K. Bhalla, F. Hirata, A. K. Rishi, and C. G. Gairola, "Cigarette smoke, inflammation, and lung injury: a mechanistic perspective," Journal of Toxicology and Environmental Health. Part B, Critical Reviews, vol. 12, no. 1, pp. 45-64, 2009.

[45] K. B. Pena, C. O. Ramos, N. P. Soares et al., "The administration of a high refined carbohydrate diet promoted an increase in pulmonary inflammation and oxidative stress in mice exposed to cigarette smoke," International Journal of Chronic Obstructive Pulmonary Disease, vol. 11, pp. 3207-3217, 2016.

[46] Y. Li, J. Yao, C. Han et al., "Quercetin, inflammation and immunity,” Nutrients, vol. 8, no. 3, p. 167, 2016. 
[47] P. P. Barros, G. H. d. Silva, G. M. S. Gonçalves, J. C. Oliveira, L. G. Pagnan, and L. Arco-e-Flexa, "Hepatoprotective effect of quercetin pretreatment against paracetamol-induced liver damage and partial hepatectomy in rats," Brazilian Archives of Biology and Technology, vol. 60, 2017.

[48] A. Ozkan, K. Fiskin, and A. Ayhan, "Effect of vitamin E and selenium on antioxidant enzymes in brain, kidney and liver of cigarette smoke-exposed mice," Biologia, vol. 62, no. 3, pp. 360-364, 2007.

[49] T. Ramesh, C. Sureka, S. Bhuvana, and V. H. Begum, "Sesbania grandiflora diminishes oxidative stress and ameliorates antioxidant capacity in liver and kidney of rats exposed to cigarette smoke," Journal of Physiology and Pharmacology, vol. 61, no. 4, pp. 467-476, 2010.

[50] A. S. Dias, M. Porawski, M. Alonso, N. Marroni, P. S. Collado, and J. Gonzalez-Gallego, "Quercetin decreases oxidative stress, NF-kappaB activation, and iNOS overexpression in liver of streptozotocin-induced diabetic rats," The Journal of Nutrition, vol. 135, no. 10, pp. 2299-2304, 2005.

[51] M. P. Gupta, K. L. Khanduja, and R. R. Sharma, "Effect of cigarette smoke inhalation on antioxidant enzymes and lipid peroxidation in the rat," Toxicology Letters, vol. 41, no. 2, pp. 107114, 1988.

[52] S. Baskaran, S. Lakshmi, and P. R. Prasad, "Effect of cigarette smoke on lipid peroxidation and antioxidant enzymes in albino rat," Indian Journal of Experimental Biology, vol. 37, no. 12, pp. 1196-1200, 1999.

[53] K. K. D. Campos, G. R. Araújo, T. L. Martins et al., “The antioxidant and anti-inflammatory properties of lycopene in mice lungs exposed to cigarette smoke," The Journal of Nutritional Biochemistry, vol. 48, pp. 9-20, 2017.

[54] E.-M. Park, Y.-M. Park, and Y.-S. Gwak, "Oxidative damage in tissues of rats exposed to cigarette smoke," Free Radical Biology and Medicine, vol. 25, no. 1, pp. 79-86, 1998.

[55] E. T. Olayinka, A. Ore, O. Adeyemo, O. Ola, O. Olotu, and R. Echebiri, "Quercetin, a flavonoid antioxidant, ameliorated procarbazine-induced oxidative damage to murine tissues," Antioxidants, vol. 4, no. 2, pp. 304-321, 2015.

[56] X. Chen, "Protective effects of quercetin on liver injury induced by ethanol," Pharmacognosy Magazine, vol. 6, no. 22, pp. 135-141, 2010.

[57] A. Helen and P. L. Vijayammal, "Vitamin C supplementation on hepatic oxidative stress induced by cigarette smoke," Journal of Applied Toxicology, vol. 17, no. 5, pp. 289-295, 1997.

[58] K. Husain, B. R. Scott, S. K. Reddy, and S. M. Somani, "Chronic ethanol and nicotine interaction on rat tissue antioxidant defense system," Alcohol, vol. 25, no. 2, pp. 89-97, 2001.

[59] F. S. Bezerra, S. S. Valença, K. M. Pires et al., "Long-term exposure to cigarette smoke impairs lung function and increases HMGB-1 expression in mice," Respir Physiol Neurobiol, vol. 177, no. 2, pp. 120-126, 2011.

[60] S. C. Lu, "Regulation of hepatic glutathione synthesis: current concepts and controversies," The FASEB Journal, vol. 13, no. 10, pp. 1169-1183, 1999.

[61] S. C. Lu, "Glutathione synthesis," Biochimica et Biophysica Acta, vol. 1830, no. 5, pp. 3143-3153, 2013.

[62] D. Xu, M.-J. Hu, Y.-Q. Wang, and Y.-L. Cui, “Antioxidant activities of quercetin and its complexes for medicinal application," Molecules, vol. 24, no. 6, p. 1123, 2019.

[63] M. P. Bindu and P. T. Annamalai, "Combined effect of alcohol and cigarette smoke on lipid peroxidation and antioxidant sta- tus in rats," Indian Journal of Biochemistry and Biophysics, vol. 41, pp. 40-44, 2004.

[64] D. DiMeo, J. Tian, J. Zhang, S. Narushima, and D. J. Berg, "Increased interleukin-10 production and Th2 skewing in the absence of 5-lipoxygenase," Immunology, vol. 123, no. 2, pp. 250-262, 2008.

[65] A. Saxena, S. Khosraviani, S. Noel, D. Mohan, T. Donner, and A. R. A. Hamad, "Interleukin-10 paradox: a potent immunoregulatory cytokine that has been difficult to harness for immunotherapy," Cytokine, vol. 74, no. 1, pp. 27-34, 2015.

[66] T. Musso, P. Cappello, S. Stornello et al., "IL-10 enhances CCL2 release and chemotaxis induced by CCL16 in human monocytes," International Journal of Immunopathology and Pharmacology, vol. 18, no. 2, pp. 339-349, 2016.

[67] I. Shachar and N. Karin, "The dual roles of inflammatory cytokines and chemokines in the regulation of autoimmune diseases and their clinical implications," Journal of Leukocyte Biology, vol. 93, no. 1, pp. 51-61, 2013.

[68] J. Zhang, T. Wu, Z. Hu, and D. Qian, "Inhibitory effect of quercetin on tumor necrosis factor and interleukin-1 beta pro-osteoclastic activities," Acta Pharmacologica Sinica, vol. 17 , no. 3, pp. 261-263, 1996. 